\title{
Improving 2D Microwave Imaging Using Known Background Properties
}

\author{
Xiande Cao, Caicheng Lu \\ University of Kentucky, Lexington, KY, 40504 \\ E-mail: xcao2@engr.uky.edu
}

\section{Introduction}

In this paper, we consider the problem of obtaining the two-dimensional (2D) image of an object by processing signals from a receiver that scans around the object. The excitation sources are planes waves generated by transmitters on the same plane as the receivers. The cross section of the object on the scanning plane is characterized by a two-dimensional distribution $g(x, y)$. This distribution is termed as the reflectivity density function. High resolution microwave imaging can be obtained using focused synthetic aperture processing [1]. In focused process, measured data is mapped onto the spatial spectrum domain, or the kspace [2]. And a two dimension Fourier transform relationship is established between spatial spectrum domain and object image. To obtain the two dimensional image, a 2D inverse Fourier transform is needed. When the spatial domain is electrically large, 2D Fast Fourier Transform (2D-FFT) can be utilized to speed up the process. In practical applications, the measured data are usually not on equally spaced grids in rectangular coordinate which is required to perform 2D FFT. Based on the different transmitter and receiver configurations, different spectrum data pattern are obtained. In this paper, we apply an interpolation scheme to obtain evenly spaced data in spectrum domain. It covers both monostatic and bi-static cases.

In certain applications, the objects of interest are often confined within a complex background. When the background scattering is relatively strong it may be difficult to produce any useful images. One such example is the through wall imaging reconstruction. In this case, the received field can be viewed as made of three components: (a) the reflection from the wall, (b) the scattering by the objects inside, and (c) the component due to the interaction of the wall and the objects. The walls generate strong reflection and the small objects inside the walls have very small contribution to the total scattered field. This leads to poor image quality for the purpose of identifying the objects inside the wall. If the property of the wall is known of partly known, it is possible to pre-calculate the scattered field by the walls only and subtract it from the received total field. If the interaction of the objects with the wall is small, then it is possible to reconstruct the object image using the difference field. The success of this treatment depends on two conditions: (a) the wall property is known (thickness, construction material, and locations), (b) the received field is accurate in both amplitude and phase. In this paper, we present simulation results based on assuming the above two conditions are satisfied. The data used for simulation are generated by hybrid 
surface-volume integral equation solver (multilevel fast multiple algorithm is applied in cases of electrically large walls).

\section{Formulations}

The 2D high resolution radar imaging using Focused Synthetic Aperture Processing (FSAP) is discussed in [1] in detail for mono-static cases. According to [1], a 2D Fourier transform pair is established between $G(X, Y)$ and $g(x, y)$, where $G(X, Y)$ is the received scattering field at spatial spectrum domain and $g(x, y)$ is the image which is termed as reflectivity density. Measured data at different frequency $f$ and incident angle $\theta$ is mapped to the spatial spectrum domain by

$$
X=(2 f / c) \cos \theta, \quad Y=(2 f / c) \sin \theta
$$

Similarly, 2D microwave imaging using FSAP can be achieved from bi-static measurement. It can also be derived from diffraction tomography using the firstorder Born approximation [2]. According to the imaging geometry in Fig. 1, the map from the object image to the scattered field can be written as,

$$
G\left(f, \theta_{t}, \theta_{r}\right)=\int_{-\infty}^{\infty} \int_{-\infty}^{\infty} g(x, y) e^{j 2 \pi\left(r_{t}+r_{r}\right) / \lambda} d x d y
$$

Under far-field approximation, the above can be rewritten as,

$$
\widetilde{G}(X, Y)=\int_{-\infty}^{\infty} \int_{-\infty}^{\infty} g(x, y) e^{-j 2 \pi(X x+Y y)} d x d y
$$

where $\beta=\theta_{r}-\theta_{t}$ is the bi-static angle, and

$$
\begin{aligned}
& X=\frac{2 f}{c} \cos \left(\frac{\beta}{2}\right) \cos \left(\frac{\theta_{t}+\theta_{r}}{2}\right), \quad Y=\frac{2 f}{c} \cos \left(\frac{\beta}{2}\right) \sin \left(\frac{\theta_{t}+\theta_{r}}{2}\right), \\
& \widetilde{G}(X, Y)=G\left(f, \theta_{t}, \theta_{r}\right) e^{-j 2 \pi\left(R_{t}+R_{r}\right) / \lambda}
\end{aligned}
$$

As analyzed above, 2D fast Fourier transform can be utilized for image reconstruction. In practice, measured data has patterns other than evenly spaced in rectangular coordinate system. For the configuration where both receivers and transmitters are located on a circle around the object, the recorded data will also be points on full or partial circles in the X-Y plane. Therefore, interpolation schemes are needed to obtain evenly distributed data samples in $\mathrm{X}$ and $\mathrm{Y}$ directions in spectrum domain.

Consider the background and the object as different scattering sources, by ignoring the interaction between two sources, the total field can be written as

$$
\mathbf{E}^{\text {total }}=\mathbf{E}^{i n c}+\mathbf{E}_{b g}^{s c a}+\mathbf{E}_{o b j}^{s c a}
$$

where $\mathbf{E}_{b g}^{s c a}$ is the scattered field due to the background and $\mathbf{E}_{o b j}^{s c a}$ is the scattered field of the objects. In the cases that $\mathbf{E}_{b g}^{s c a}$ is high, the objects may be undetectable. In cases when the background properties are known, it is possible numerically calculate the scattered field (of the same source) by background only. If the background scattered field is subtracted from the total measured field, it is 
expected that better reconstruction of the objects could be achieved. In the following, we present image reconstruction results to verify the interpolation scheme and demonstrate the improvement using background subtraction method. The scattered data for the imaging processing are from the solution of the forward scattering problem using the method of moment.

\section{Computational Results}

To validate the interpolation schemes and image improvement, intensive computational simulation has been performed. In our simulation, the scattered field is calculated by method of moment (using the hybrid volume-surface integral equation). Figure 2 shows the image results of three metal spheres in free space with two configurations: (a) mono-static and (b) bi-static. Frequency samples are equally spaced at $0.8 \mathrm{GHz}$ to $1.0 \mathrm{GHz}$. The number of incident angle is 61 for both cases. And the number of receiver for bi-static is also 61. All angle coverage is $[0,2 \pi]$. Spheres are located at $(-0.1,0),(0.1,0)$, and $(0,0.16)$ (unit is meter). In Figure 3, (a) is the geometry of the image objects which consists of fives spheres in an enclosed wall. The dimension of the wall is $16.8 \mathrm{~m} \times 8.4 \mathrm{~m}$ with thickness $0.225 \mathrm{~m}$. The number of incident angle is 91 and the number receiver is 121 , both scan in the $[0,2 \pi]$ angular range. The frequency is $300 \mathrm{MHz}$. Spheres are located at $(0,0),(2.52,2.52),(-2.52,2.52),,(-2.52,-2.52$,$) , and (2.52,-2.52)$. Figure (b) shows that the five spheres are hidden by the background. By subtraction, (c) shows the improved image of those spheres.

\section{Conclusion}

This paper presented numerical simulation of imaging reconstruction of objects confined within a dielectric wall. The image for the unknown region can be improved if the scattering properties of the wall are known. This improvement is achieved by subtracting the scattered field of the wall. From the total scattered field. The scattered field for wall alone case is obtained by solving a forward scattering problem. Whether this approach can be applied to the partially known background needs to be further investigated.

\section{References}

[1] Mensa, Dean L., High Resolution Radar Imaging,. Dedham: Artech house, 1981.

[2] Chew, Weng Cho, Waves and Fields in Inhomogeneous Media, New York: Van Nostrand Reinhold, 1990

[3] Berizzi, Fabrizio, and Giovanni Corsini, "A New Fast Method for the Reconstruction of 2-D Microwave Images of Rotating Objects," IEEE Trans on Image Proc, vol. 8, no. 5 (1999): 679-687.

[4] Marion, Dominique, "Processing of ND NMR spectra sampled in polar coordinates: a simple Fourier transform instead of a reconstruction," Journal of Biomolecular NMR, (July 2006): 45-54. 
[5] Press, William H., Teukolsky, Saul A., Vetterling, William T., and Flannery, Brian P., Numerical Recipes The Art of Scientific Computing, 3rd ed. New York: Cambridge University Press, 2007.

[6] Keller, J. B., "Geometrical theory of diffraction," J. Opt. Soc. Am. 52 (1962): 116-130.

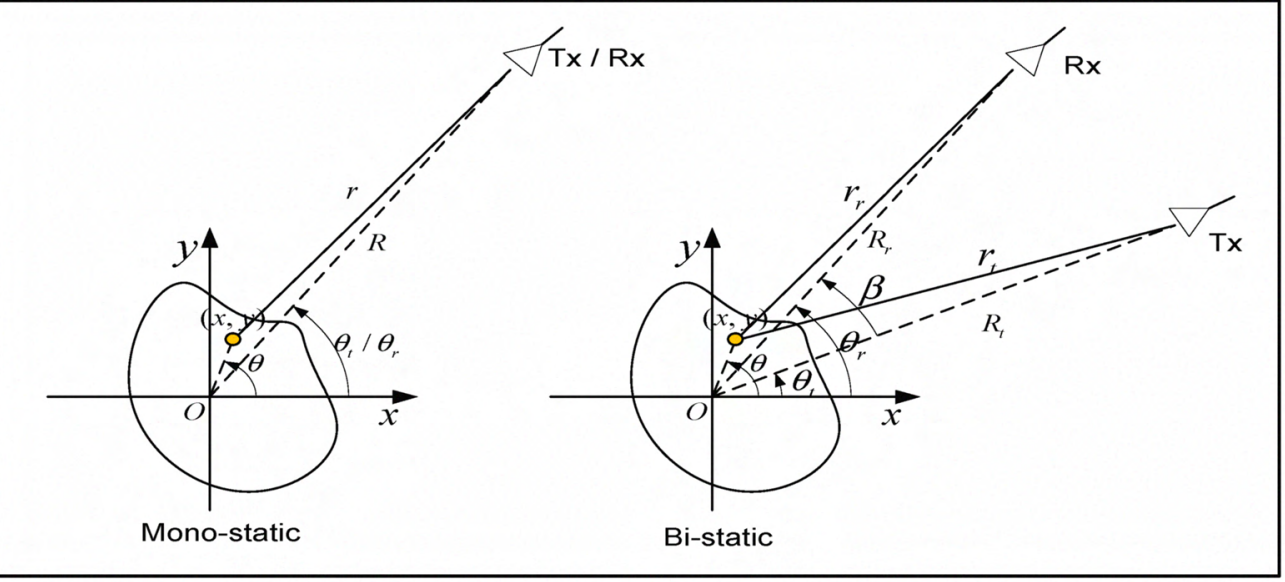

Figure 1. Mono-static and Bi-static imaging geometries.

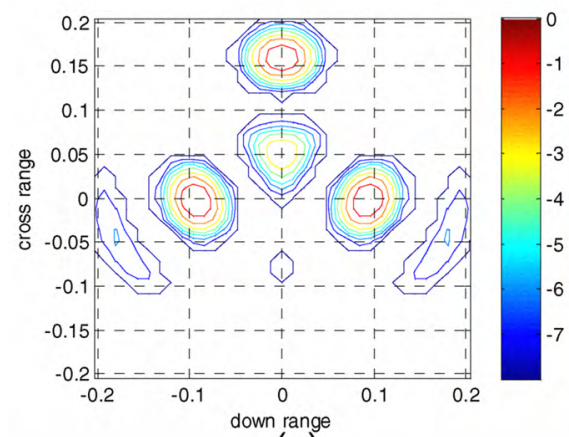

(a)

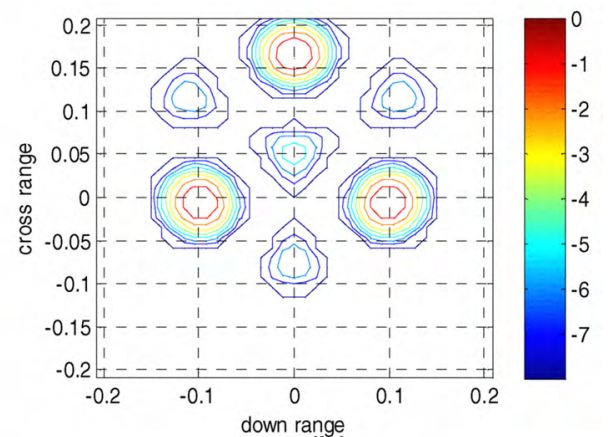

(b)

Figure 2. Mono-static and Bi-static images for three spheres in free space

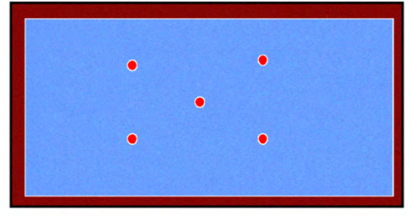

(a)

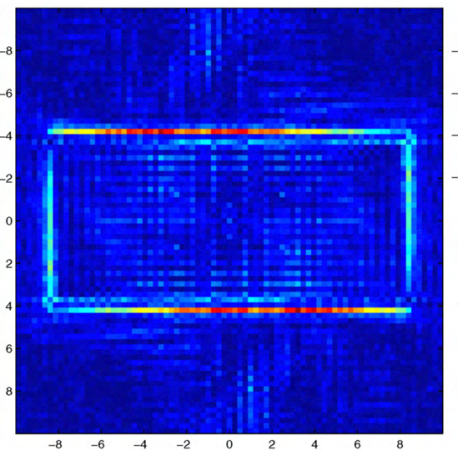

(b)

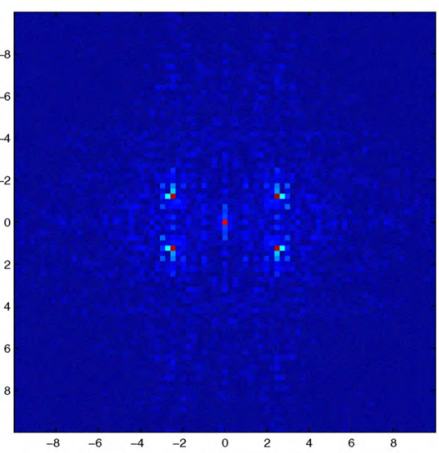

(c)

Figure 3. Image of five spheres in an enclosed wall: (a) the original objects and wall, (b) the image reconstructed using total scattered field, (c) the image reconstructed using residue field. 